\title{
A Future Program of Muon Scattering at the Tevatron
}

The Tevatron Muon Collaboration

March 51988

In this document we outline a program of Muon Scattering Physics at the Fermilab Tevatron which goes beyond that possible with the currently installed E665 apparatus. 


\section{Introduction}

The E665 experiment was proposed in 1980/81 [1] and recently completed its first period of data taking. At this stage it is appropriate to review the directions of the program which was always intended to cover many periods of data taking.

In the first section below we summarise the data taken during the period and attempt to characterise the physics results which we hope to achieve with that data.

We do not anticipate major upgrades before the next run. We describe, in the second section below, a program of measurements which we expect to make in that running period.

We then itemise briefly the future physics which is possible with the excellent muon beam and a modified apparatus. This is in four parts, two of which involve moderate upgrades to the apparatus. At the present time both of these options appear very attractive to the collaboration and we intend to pursue both at this stage. The timescale to implement each is such that testing of apparatus towards the end of the next run is a possibility.

From our point of view, it is very desirable that the PAC reacts to our plans so that more intense attempts to organise funding may be made. It should be pointed out that as well as the two funding agencies in the US, two in Germany will also be involved. Preliminary support has been obtained already but this, without some indication that approval is possible, is understandably modest. 


\section{Data Taken 1887/88}

Although some fraction of many detector components had been present for the 1985 Beam Test, it was in 1987 that the detector was completely commissioned. First beam appeared in the New Muon lab in July 1987 and the muon beam was rapidly re-established. Preliminary detector tuning and calibration followed during July and some of August before the accent changed to a series of studies to establish a range of suitable triggers. This work was complete by mid-September and Data Taking commenced on October 6, 1987, with all detectors except the Ring Imaging Cerenkov counter (RICH) and the Wide Angle Proportional tubes fully operational. The latter detectors were fully working by November 1. For the RICH counter the operation was a learning experience with tuning going hand in hand with the data taking. The complete detector is shown in Fig. 1.

The muon beam operated well; a tune of $500 \mathrm{GeV}$ muons was chosen: this is essentially the maximum energy tune available with $800 \mathrm{GeV}$ protons from the Tevatron. The effective yield for this tune was $0.55 \times 10^{-5} \mu /$ proton.

The triggers established along with their rates are itemised in Table I. The two primary physics triggers are the Large Angle Trigger (LAT) which is sensitive down to a muon scattering angle of 3-4 mr and the Small Angle Trigger(SAT) which is sensitive down to about $0.5 \mathrm{mr}$. The SAT selects a $12 \%$ fraction of the beam used for the LAT and projects the individual beam trajectory, as defined by the Beam Hodoscopes, to the rear of the apparatus. There the absence of an un-scattered muon generates the trigger. As operated, both the LAT and SAT contained dominantly non-target associated triggers; however, the rates were low enough that the effective dead time did not exceed $15 \%$. A summary of the data taken with different targets and at different beam energies is given in Table II. The kinematic regions accepted by the Triggers are shown in Fig. 2. It is worth mentioning that the data on both Hydrogen and at low energy on Deuterium were only possible as a result of the extensions of the run to February 15, 1988.

The Streamer Chamber has a longer associated dead time than the electronic readout, so an additional constraint to the trigger was applied; this required a signal from the $z$ planes of the PC proportional wire chambers which was consistent with at least one particle outside of a $\pm 96 \mathrm{~mm}$ horizontal band. Monte Carlo studies indicate that this trigger is 
close to $100 \%$ efficient for a hadronic center of mass energy squared greater than $150 \mathrm{GeV}^{2}$. A summary of the Streamer Chamber data is given in Table III. The total measurement capacity in the group is $20 \mathrm{~K}-30 \mathrm{~K}$ pietures per year. It is intended that a selection of the interesting pictures will be made on the basis of the analysis of the spectrometer data. An initial possibility would be to select only the very highest hadronic energy data.

As indicated in the tables, data were taken with Deuterium, Xenon and Hydrogen targets. The luminosity of the Deuterium data is reapectable, and it is hoped that considerable advances will be made in understanding the QCD structure of the hadronic final state. The data from the other two targets will permit us to make investigations of both A-dependent and Non-singlet phyrics. Both are especially interesting in the new kinematic regimes available with the Tevatron. In addition the low energy data is expected to yield interesting data at very low $Q^{2}$ and to permit study of formation and fragmentation lengths in the transition region between SLAC and the higher energies. An E665 typical event is shown in Fig. 3. 


\section{Run Plan for the 1989 Run}

In sereral respects the data taken in $1987 / 8$ is exploratory. Although the apparatus worked very well, the data, taken on the different targets, were in rather long blocks, leaving them vulnerable to time dependent systematic effects. On the other hand the Streamer Chamber data set is rather large.

We have therefore decided to focus the second run on achieving a well controlled set of data on targets with a range of $A$ values in order to study in some detail the behaviour of both the Structure Functions and the hadron production as A is varied. The goal is to use the nuclenr targets to provide intrinsic length scales for QCD phenomena, both for the static distributions in structure functions, and static and dynamic effects in hadron production and hadronization. We are currently studying various possible target configurations which would minimise systematic effects in ratio measurements. The advantages of concurrent running with several targets separated along the beam direction is being contrasted with the technique of frequently interchanging targets. In any case it is clear that the reference nucleus will be Deuterium.

We are conscious of the possibility that Deuterium may itself be special. We therefore anticipate some running with a Bydrogen target. This running will then also be a logical next step in understanding the systematic errors associated with a fully thought out attack on 'the definitive structure function measurement'. As far as the hadron work is concerned, the RICH counter will be fully operational and the rery interesting questions associated with the leading hadron production will be fully addressable.

Apart from modification of the target configuration, most of the improvements will be in the triggering of the apparatus.

i) A LAT trigger based on target pointing with the proportional tubes at the rear of the apparatus will discriminate against scattering in the hadron absorber and the calorimeter. This would enrich the LAT data by a factor of about 4.

ii) The SAT will be enhanced to give it sensitivity to about $70 \%$ of the beam which is used for the LAT. This is both an enrichment of the SAT data and an enhancement of effective luminosity by a factor 5 . 
These improvements will permit some increase in the beam intensity. In addition we do not expect as long a tune up period as during the first run. We would therefore expect the run (about 6 months) to yield an effective integrated flux of about $(3-4) \times 10^{12}$ muons at $500 \mathrm{GeV}$. This clearly depends on the proton allocation to the experiment. We imagine that our request will exceed $6 \times 10^{12}$ protons per pulse. Achievement of such an integrated muon flux represents an increase of about a factor 4 over the 1987-88 run.

The effective muon luminosity will be higher if we spend some time at lower energies. This is probably necessary to ensure that we understand the influence of $R=\sigma_{L} / \sigma_{T}$ on our structure function ratios. $500 \mathrm{GeV}$ is the maximum mean beam momentum practical with the Tevatron operating at $800 \mathrm{GeV}$. If the operating energy of the Tevatron were increased we would increase our beam momentum proportionately.

A short Streamer Chamber run may be desirable and this could be accommodated. 
Future Program beyond the 1989 Run

1. High Statistics Badron Production, QCD and Fragmentation.

a) Physics Motivation

The motivations for study of hadron production by muons at Teratron energies are several, the relevant quark model diagram and the lowest order QCD corrections are illustrated in Fig 4.

i) The energies involved are high enough that the QCD structure of the interaction should be evident. The energy is comparable with the energies at PETRA and PEP and furthermore the current direction is well defined by the measurement of the incident and scattered muons.

ii) A direct comparison with $e^{+} e^{-}$interactions, (both interactions hare a virtual photon), is interesting beyond any issues of currently popular models.

iii) Whereas $e^{+} e^{-}$interactions are democratic, (all generations and flavours of quark contribute in proportion to the square of the quark charge), the nature of the interaction in muon scattering may be tuned by choosing the $x_{b j}$ varisble. This applies in both the simple quark model and at the level of first order QCD corrections. At low $x_{b j}$ the photon gluon fusion graph should dominate, at higher $x_{b j}\left(x_{b j}>0.1\right)$ the quark-gluon bremsstrahlung graph dominates. This should manifest itself in the badrons. The interaction should therefore provide a fertile laboratory for the study of the hadronic nature of the fragmentation of different partons.

iv) The backward $\left(x_{f}<0.0\right)$ region involves the fragmentation of the target remnants, this may be a diquark aystem or it may be a 'colored proton'.

The earliest high energy $\mathrm{e}^{+} e^{-}$data sets were of the order of sereral tens of thousands of events, that is also the level of statistics obtained by the EMC experiment[2] using a Streamer Chamber, it is also the size of data set possible in a two year analysis of the current E665 Streamer Chamber data. These data sets are not sufficiently rich to permit extenaive study of the more particular elements of fragmentation. A good example is Baryon-antibaryon production. Even with current $e^{+} e^{-}$data samples approaching $10^{5}$ events the yield is not high. 
b) Modification to the Experiment, Time Projection Chamber.

We propose to replace the Streamer Chamber with a Time Projection Chamber. The TPC would be situated in the Vertex magnet. Such a possibility was considered earlier[3] for muon experiments at CERN however recognition of the existence of E665, with higher energy, meant that that possibility was not pursued. In the context of E665 the approach was rejected on the basis of the then projected running schedule.

c) Advantages of a TPC

The advantages of this approach are:

i) The dead time is reduced by a large factor as compared to the Streamer Chamber dead time of 200-500 millisecs.

ii) An improvement in analysis time. Even allowing 1 year for the development of analysis code the analysis of a sample of many hundreds of thousands of events, within 18 months of data taking, should be feasible.

iii) The readout from both wires and pads in the TPC can give good particle identification as illustrated in Fig. 5. Such particle identification has previously never been possible in a deep inelastic lepton scattering experiment in the target fragmentation region.

iv) The analysis of Streamer Chamber pictures involves large costs associated with manpower. The analysis of the TPC data involves none of these costs.

d) Implications, Implementation of the TPC.

A sketch of the proposed Time Projection Chamber is shown in Fig. 6., Fig. 7. shows the proposed layout of pads on the TPC end-plates. A view of a Monte Carlo generated event seen in the TPC is shown in Fig. 8. It should be noted that currently it is thought to be necessary to 'deaden' the beam region. The pattern of pads hit in a single event is illustrated in Fig. 9 along with the distribution of the number of significant hits per event. Halo tracks and noise hits are not included.

A detailed proposal is in preparation and will be available within some weeks, a preliminary set of specifications is given in Table IV. There are several comments which may already be made. 
i) The TPC as a detector is now well understood[4] and the successful progress in construction of TPC detectors for large collider environments has resolved several questions that remained open in 1983. There has been a recent proposal[5] for a TPC with a similar scale to that considered here which has to operate in the high multiplicity conditions of heary ion collisions.

ii) The MPI-Munchen laboratory has considerable expertise having participated in the construction of the Aleph TPC[6]. In general we feel that there is ample expertise to permit us to manufacture the field cage and detectors, including end-plates before final assembly at Fermilab.

iii) The choice of detectors, MWPC's with anode wire and cathode pad readout is designed to provide excellent spatial resolution and adequate two-track separation. The latter is limited by both the operation of the MWPC and by the electronics.

iv) The total number of electronic channels is about 6000 including wires and pads. Conventional electronics using Flash ADCB implies a total system cost of about $\$ 200$ per channel. Modern semi-custom work suggests that the more relevant cost range may be $\$ 100$ per channel. Giren the small number of channels, as compared to 150,000 for a typical LEP experiment TPC there are clear advantages to using an existing implementation.

v) The magnetic field of the vertex magnet has a certain degree of non-uniformity because the return yole has been minimised to provide as wide as possible angular acceptance for the existing detector. This would not be necessary for the TPC and there may be considerable advantages to haring a more uniform field. We may therefore propose to introduce a return yoke on the downstream side of the magnet similar to that currently in place on the upatream side. The modular design of the CVM would facilitate this.

vi) The timeacale for production of the TPC is of the order of 2 years. Given the current schedule this puts it bejond the next run, however, if approval and funding are available by the Autumn of 1988 it is possible that a teat of the detector could be made in the Tevatron Muon beam at the end of that run. 


\section{e) Summary}

Taking into account the factors above, the collaboration is enthusiastic about both the physics and the feasibility of the detector. As mentioned earlier, a detailed proposal, including extensive cost and manpower estimates, is in preparation. 


\section{Charged Current and other High Luminosity Studies}

In Spring 1987, we submitted a Letter of Intent[7] to the PAC to alert them to the fact that we were considering a program of measurements with a Target Calorimeter. A very similar program of Physics was discussed in the Workshop on Cern SPS Fixed Target Physics[8].

a) Phyaics Motiration

i) Weak Charged Current Physics

The primary motivation for a high luminosity calorimeter target is that it permits studies of the Weak Charged Current interaction. The helicity of the incident lepton, the muon, is known in the polarised mode of the Tevatron Muon Beam[9]. This means that the muon polarisation can be chosen so that the normal charged current interaction is forbidden and there is a sensitivity to the existence of a higher mas right handed weak boson. It appears that a sensitivity to the Right Handed Boson exchange for boson masses up to $400-500 \mathrm{GeV}$ is achievable.

This is comparable with the most respected low energy searches[10]. These measurements are necessarily restricted in the other parameters of the interaction. In muon decay experiments this restriction is often expressed as an upper limit on the mass of the associated right-handed neutrino to which the experiment is sensitive.

The measurement is also proposed[11] for HERA, assuming that polarisation of the electrons in that machine is schieved.

An important systematic exror is contributed by the uncertainty in the polarisation of the muons. However it is not the polarisation of the individual muons which is important but that of the ensemble. The systematic error on that quantity is $\mathbf{0 . 0 2}$.

ii) Other High Luminoxity Phyries.

The excellent calorimeter and muon detection necessary to perform the Charged Current measurements implies that a whole program of high lumincaity multi-muon measurements is possible. These include $\tau^{+} \tau^{-}$production, $J / \psi$ production, Upsilon production and multi-muon production that indicates heary quark production. The lominosity is such 
that, in a typical run, there should be a yield of about 1000 events in which both $B$ and $\bar{B}$ hadrons decay semi-leptonically to muons and both muons are detected. This would give an initial handle on the mixing.

b) Modification to the experiment, Calorimeter Target.

The key to detection of the Charged Current Interaction, which is a prerequisite if one wants to learn angthing about the possibility of right-handed currents, is the detection and measurement of the total hadronic vector event by event. Using the incident muon vector along with the hadron vector gives a prediction of the direction and momentum of the outgoing lepton, muon or neutrino. If there is a muon it will be detected as predicted, if it is a neutrino it will be absent. Previous calorimeter targets used in muon scattering[12,13] have achieved good hadronic energy resolution but have not addressed the question of hadron direction. We propose to use an $8 \mathrm{~m}$. long Uranium Scintillator calorimeter which could have a flucial mass of about $3000 \mathrm{gm}-\mathrm{cm}^{-2}$.

c) Implementation of the Calorimeter Target

Our current design[7] is illustrated in Fig. 10. It is modular in design, a single module is about $80 \mathrm{~mm}$ in the beam direction and $400 \mathrm{~mm} \times 400 \mathrm{~mm}$ transverse to the beam. The individual sheets of Uranium are $3 \mathrm{~mm}$ but this is not yet flred.The scintillation light generated in each module is read out on each side of the module and the differential of the light output determines the traneverse powition of the centroid of the hadron shower. A series of these measurements then determines the hadronic angle. A sketch of the muon scattering binematics with the hadronic parameters superimposed is shown in Fig. 11. Studies with Monte Carlo calculations of hadron showers, and experiments with test scintillators and wavelength ahifters suggest that the resolution in angle can be (25 mrad $/ \sqrt{E}$ ) and the resolution in energy $(40 \% / \sqrt{\bar{E}})$.

The Errors in the $Q^{2}-\nu$ plane associated with an angle resolution of $2 \mathrm{mrad}$. are shown in Fig. 11. It is clear that this limits the range of binematics for which the charged current can be detected in a background of misidentified neutral current ( photon exchange) events. While there are indications that the calorimeter as sketched here and as described in Ref [7] is adequate, other schemes involving scintillating fibre readout are also under study. 
d) Timescales for Construction of Calorimeter Target

There have been extensive calorimeter design studies in recent years particularly with respect to current collider experiments and with respect to SSC possibilities. The field is considerably better understood than 4 years ago. The present design and cost estimate envisages using depleted Uranium plates, but other high density, high $\mathrm{Z}$ materials such as Tungsten can be used. We are closely monitoring the test results of other calorimeters, and in particular a calorimeter designed by ORNL for a CERN experiment, which has a similar readout from both ends of a scintillator using wavelength shifting bars. Preliminary results of a year ago tend to confirm the calculations, both of the energy resolution and of the direction of energy flow. This group performed a test with $200 \mathrm{GeV}$ protons in February 1988 , and the results from the run should be arailable by the end of March.

We feel that we can finalise a design during the next 6 months and proceed with construction over a period of 1 year to 18 months from then. A limited number of modules would be available during the next 1989 Fixed Target run and their operation in the muon beam can be tested with the modules initially sitting behind the E665 apparatus. If these are succesaful, further teats of a few days duration upatream of the CVM, approximately in the final target position, could be contemplated.

\section{e) Summary}

In summary there appear to be no fundamental problems which prevent construction of the Target Calorimeter which would open a fleld of measurements which have not been performed before, have a sencitivity to phyrics beyond the standard model and which are competitive with the mearurements envisaged, for a few years hence, at HERA. 


\section{Eigh Preciaion Structure Function Measurements}

Measurements of the Structure Functions of the Nucleon, particularly from the elementary target, the proton, have provided the most precise determination of the cut-off parameter, $\Lambda_{Q C D}$, of the strong interaction. The previously obtained accuracy has been typically a few percent[14]. Provided that aufficient luminosity can be obtained, which implies targets of 6 metres or longer, the determination is dominated by systematic effects. Some of these are associated with radiative corrections, some with the influence of $\mathrm{R}=\sigma_{L} / \sigma_{T}$, some with the knowledge of the incident and scattered muon energies, some with the reconatruction efficiency and some with the beam counting.

As mentioned above, the advertised precision of the $F_{2}$ measurements is a fer percent however the current status[15] is that there is a discrepancy of up to $25 \%$ between two of these measurements. We expect that with the data obtained in the past and next runs we will be able to make some statement about this discrepancy. On the other hand we do not feel that a measurement which does not take special measures to address the determination of $F_{2}$ at better than $1 \%$ systematic accuracy will contribute to the determination of $\Lambda$. We therefore plan, during the next run, to study and steadily reduce the systematic error in our measurements of crose-section with the intention of reaching this $1 \%$ level of preciaion. We therefore defer a long target and a direct attack on this interesting subject while recognising that if the understanding of the current data does not improve over the next two years, such an attack becomes almost imperative.

\section{Polarised Structure Funetions}

Finally we mention the subject of the Polarised Structure Functions. These have been recently meanred[16] uring the muon beam at Cern with a 1 metre long double polarised target. The results are extremely interesting since they are, at first glance, contrary to expectations. The results suggest, either that there should be significant nonzero polarisation effects in scattering from the Neutron,or (and??) that the polarisation of a nucleon does not reaide on the quarks. At this stage we do not include such measurements in our program because there are already two initiatives[17] in progress to addreas these issues. One uses an internal target in the electron ring at HERA and the other uses the muon beam at CERN. Both these initiatives involve members of our collaboration. 


\section{Conclusion, Request to the PAC}

In conclusion we ask the PAC to consider the program as outlined above and to react to the Physics of the measurements, especially those with the TPC and with the Calorimeter Target. We will request approval in Autumn 1988 for running in the two data taking periods after the 1989 run. The urgency is driven by the timescales for funding and construction. We are working on detailed proposals and will be happy to respond to any questions the PAC may have. The programs as outlined above are clearly rather full of physics and would stretch the resources of the collaboration as currently constituted. On the other hand we are not a closed collaboration and we feel that the physics issues are of such interest that we can attract further participation. There are already some preliminary indications in this direction. 


\section{References}

[1] E665 Proposal, Muon Scattering with Hadron Detection at the Teratron.

[2] EMC NA9 Data, eg M.Arneodo et al; Z. Phys. C 35,417 (1987)

M.Arneodo et al; CERN-EP/87-112(1987), submitted to Z. Phys.C

[3] H.M Braun, CERN 83-02 Vol II, pp 97-112, 1983.

[4] F. Sauli, CERN-EP/87-223 (1987)

[5] H. Wieman et al, HISS TPC Proposal(Preliminary), Jan 1988.

[6] Aleph Technical Report,CERN/LEPC/83-2(1983)

[7] Letter of Intent, Weak Interaction and Heavy Quark Physics with the Tevatron Muon Beam, Richard Wilson, April 29, 1987.

[8] CERN 83-02, Vol II, 1983, Benvenuti A.C. pp30-54. Smadja G. and Vesztergombi G. pp55-65. Clift R.W., Gossling C., Sloan T. and Wimpenny S.J. pp 83-96

[9] Malensek A. and Morfin J. BT0041 and Fermilab TM 1193, July,1983.

[10] J.Carr, Proc. XXIII Int. Conf. on High Energy Physics. Stewart C. Loken, Ed ; Berkeley 1986, p979.

[11] C.H. Llewellyn Smith, Proc. XXIII Int. Conf. on High Energy Physics.Stewart C. Loken Ed; Berkeley, 1986, p255.

[12] BFP, G.D. Gollin et al., Phys. Rev.D24 (1981) 55.

[13] EMC, J.J. Aubert et al., Nucl. Phys. B213, (1983) 1.

J.J. Aubert et al., Nucl. Phys. B213, (1983) 31.

J.J. Aubert et al., Nucl. Phys. B272, (1986) 158.

[14] EMC, J.J. Aubert et al., Nucl. Phys. B259, (1985) 189.

CHIO, B.A. Gordon et al., Phys. Rer. D20 (1979) 2645.

BCDMS, A.C. Benvenuti et al., Paper \#216 submitted to the Int. Symp. on Lepton and Photon Interactions at High Energy, Hamburg, July, 1987.

[15] Sloan T. Plenary Tall at the Int. Europhysics Conf. on High Energy Physics, Uppsala, Sweden, June 1987. CERN-EP/87-188.

Voss R. Invited tall at the Int. Symp. on Lepton and Photon Interactions at High Energies, Hamburg, July 1987.

[16] EMC, J. Ashman et al., CERN-EP/87-230, Dec. 23, 1987, submitted to Phyrics Letters.

[17] Hughes V., Geesaman D., Private Communications. 


\section{Tables}

Table I: Trigger Rates.

Table II: Summary of Data Taken in 1987/88.

Table III: Streamer Chamber Data Sets

Table IV : Preliminary Time Projection Chamber Specifications 


\section{Figures}

Fig. 1. E665 Apparatus Layout.

Fig. 2. Kinematic Regions accepted by the LAT and SAT triggers.

Fig. 3. An E665 Event.

Fig. 4. Graphs of Hadron Production by Muons.

Fig. 5. Energy Loss as measured by the Berkeley TPC.

Fig. 6. Sketch of the proposed TPC.

Fig 7. Layout of Cathode Pad Rows on the TPC end- plates.

Fig. 8. Monte Carlo event as seen in the TPC.

Fig. 9a. Pattern of pads hit by a single event.

Fig. 9b. Number of pad hits per event.

Fig. 10. Layout of proposed Target Calorimeter.

Fig. 11. Muon Scattering binematic with hadronic parameters superimposed. 
Table I

\section{E665 TRIGGER RATES $1987 / 88$}

LAT Rate per inc. Beam Muon
SAT Rate per

inc. SAT Beam Muon

$\begin{aligned} & \text { Deuterium } \\ & 500 \mathrm{GeV}\end{aligned}$
$(1.5-2.3)^{\star} 10^{* *}-5 \quad(0.8-1.0)^{*} 10^{* *}-4$
$\begin{aligned} & \text { Xenon } \\ & 500 \mathrm{GeV}\end{aligned}$

Hydrogen $500 \mathrm{GeV}$

$(1.3-1.9)^{*} 10^{* \star}-5$

$(1.3-1.7)^{\star} 10^{\star \star}-4$

Deuterium

$(1.1-1.2)^{\star} 10^{\star \star}-4$

$(1.3-1.7)^{*} 10^{* *}-4$

$100 \mathrm{GeV}$

Xenon $100 \mathrm{GeV}$

$(1.1-1.2)^{*} 10^{* *}-4$

$(4.1-4.8)^{\star} 10^{* \star}-4$ 


\section{7-88 Run Statistics}

\section{GEV MUONS}

$\begin{array}{cllll}\frac{\text { TARGET }}{\mathrm{D}_{2}} & \frac{\text { TAPES }}{1100} & \frac{\text { MUONS }}{2.5 \times 10^{11}} & \frac{\text { TRIGGERS }}{7.5 \times 10^{6} \text { LAT }} & \frac{\text { EVENTS }}{7.5 \times 10^{5}} \\ & & & 5.0 \times 10^{6} \mathrm{SAT} & 5.0 \times 10^{5} \\ \mathrm{Xe} & 650 & 1.5 \times 10^{11} & 4.5 \times 10^{6} & 2.3 \times 10^{5} \\ & & & 4.0 \times 10^{6} & 2.0 \times 10^{5} \\ \mathrm{H}_{2} & 690 & 1.5 \times 10^{11} & 3.2 \times 10^{6} & 2.3 \times 10^{5} \\ & & & 3.3 \times 10^{6} & 1.7 \times 10^{5} \\ \text { SUM } & 2440 & 5.5 \times 10^{11} & 1.5 \times 10^{7} & 1.2 \times 10^{6} \\ & & & 1.2 \times 10^{7} & 0.9 \times 10^{6}\end{array}$

100 GEV MUONS

\begin{tabular}{|c|c|c|c|c|}
\hline$\frac{\text { TARGET }}{X_{e}}$ & $\frac{\text { TAPES }}{440}$ & $\frac{\text { MUONS }}{3.5 \times 10^{10}}$ & $\begin{array}{l}\text { TRIGGERS } \\
3.5 \times 10^{6} \\
1.4 \times 10^{6}\end{array}$ & $\begin{array}{l}\text { EVENTS } \\
5.6 \times 10^{4} \\
3.5 \times 10^{4}\end{array}$ \\
\hline$D_{2}$ & 340 & $2.6 \times 10^{10}$ & $\begin{array}{l}2.6 \times 10^{6} \\
1.2 \times 10^{6}\end{array}$ & $\begin{array}{l}8.3 \times 10^{4} \\
6.0 \times 10^{4}\end{array}$ \\
\hline SuM & 780 & $6.1 \times 10^{10}$ & $\begin{array}{l}6.1 \times 10^{6} \\
2.6 \times 10^{6}\end{array}$ & $\begin{array}{l}1.4 \times 10^{5} \\
.95 \times 10^{5}\end{array}$ \\
\hline $34 \mathrm{NO}^{2}$ & 3280 & $0.08310^{119}$ & $\begin{array}{l}2.13 \times 10^{7} \\
1.32 \times 10^{7}\end{array}$ & $\begin{array}{l}1.0 \times 10^{6} \\
9.0 \times 10^{6}\end{array}$ \\
\hline
\end{tabular}


Table III

\title{
STREAMER CHAMBER DATA 1987/88
}

\author{
PICTURES PICTURES
}

with INTERACTION

$\begin{array}{lrr}\begin{array}{c}\text { Deuterium } \\ 500 \mathrm{GeV}\end{array} & 210,000 & 73,000 \\ \begin{array}{l}\text { Xenon } \\ 500 \mathrm{GeV}\end{array} & 156,000 & 55,000 \\ \begin{array}{c}\text { Hydrogen } \\ 500 \mathrm{GeV}\end{array} & 127,000 & 25,000\end{array}$

Deuterium

16,000

5,000

$100 \mathrm{GeV}$

Xenon

44,000

17,000

$100 \mathrm{GeV}$ 
Table IV

\section{Main Vertex TPC Parameters}

- height $2 \times 300 \mathrm{~mm}$

- length $1550 \mathrm{~mm}$

- width $600 / 1450 \mathrm{~mm}$ conically shaped with $42.5^{\circ}$ acceptance in the bending plane

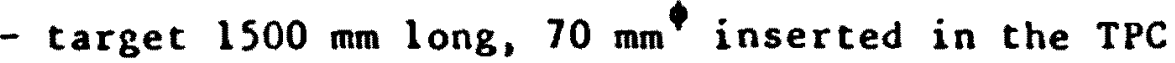

- TPC field cage $-6 \mathrm{kV}$ with beam pipe of $100 \mathrm{~mm}$

- sense wire spacing $4 \mathrm{~mm}$

- 21 pad rows with $8 \times 8 \mathrm{~mm}^{2}$ pads $\left(5 \times 8 \mathrm{~mm}^{2}\right.$ near the beam)

- row distance varying from $100 \mathrm{~mm}$ to $40 \mathrm{~mm}$

- pressure $1 \mathrm{~atm}, \mathrm{gas}$ mixture $80 \%$ Argon, $20 \%$ methane

- gate wire plane in front of the grid wire plane to avoid production of ions if no trigger takes place

- resolution: in $y \sim 250 \mathrm{~mm}$, in $z \sim 1 \mathrm{~mm}$ (with 2 to $5 \mathrm{~cm}$ drift per $\mu \mathrm{sec}$ and with a 60 nsec clock readout), in $x$ defined by $4 \mathrm{~mm}$ sense wire pitch

- readout time $\sim 15 \mathrm{usec}$

- z coordinate loss near the end plates $\sim 10 \mathrm{~mm}$ if the trigger is formed in $500 \mathrm{nsec}$

- two track separation $2 \times 2 \mathrm{~cm}^{2}$

$-\Delta p / p^{2} \sim 0.5 \%$ proportional to $\sigma_{y} / B^{2} \sqrt{N}$ with $L=$ track length and $N=$ number of pad crossings 


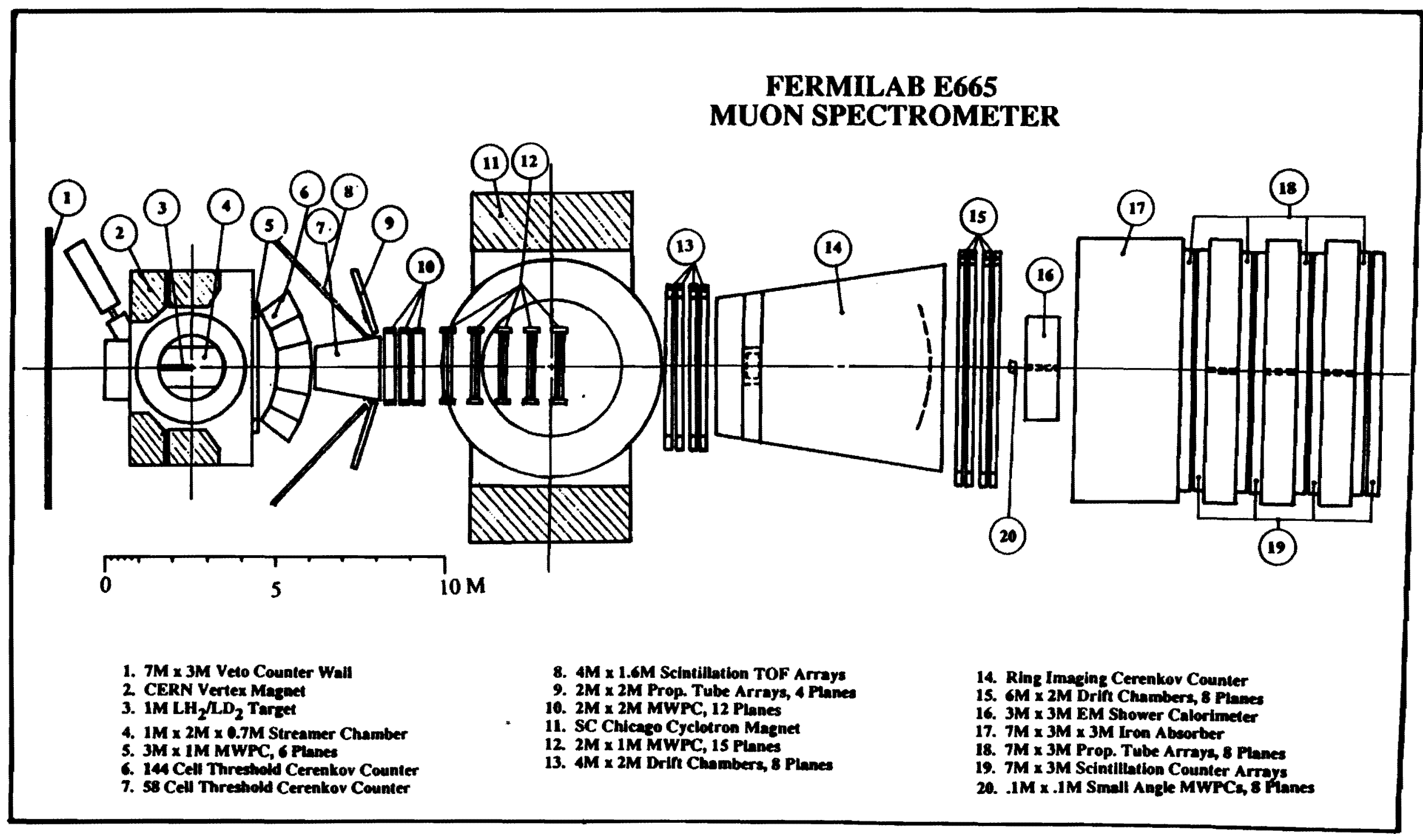

Fig. 1 


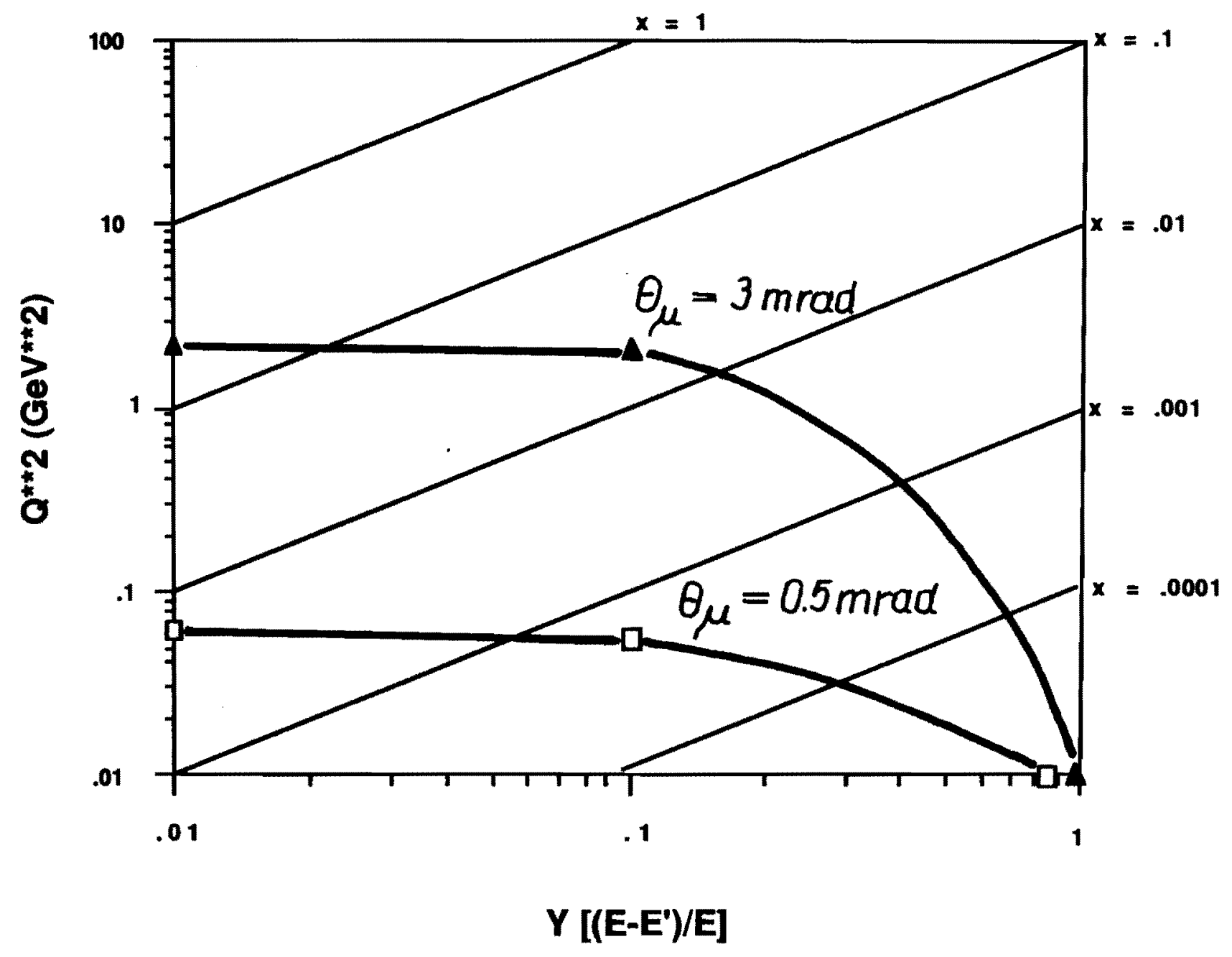

Fig. 2 


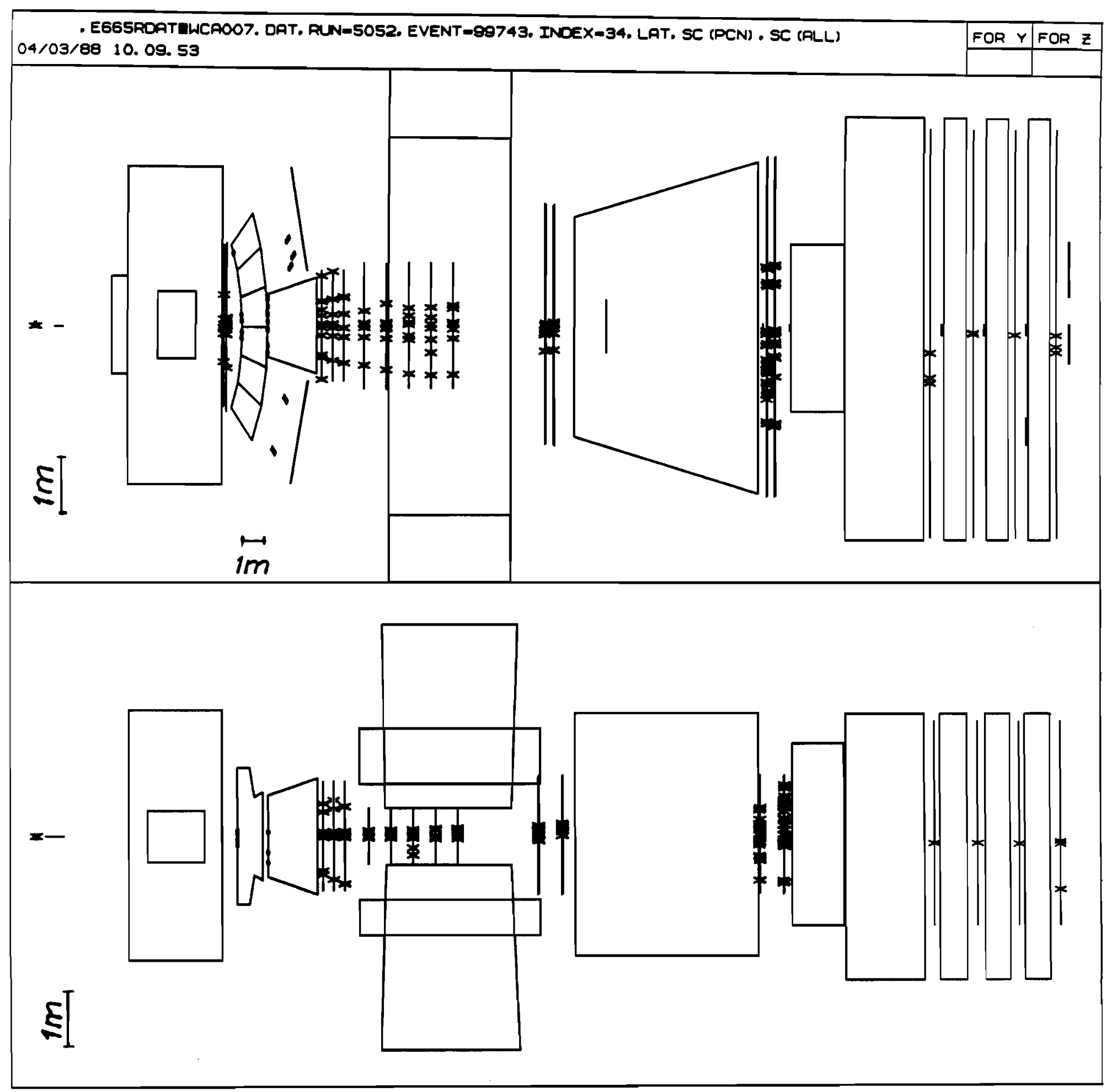

Fig. 3 
(a)

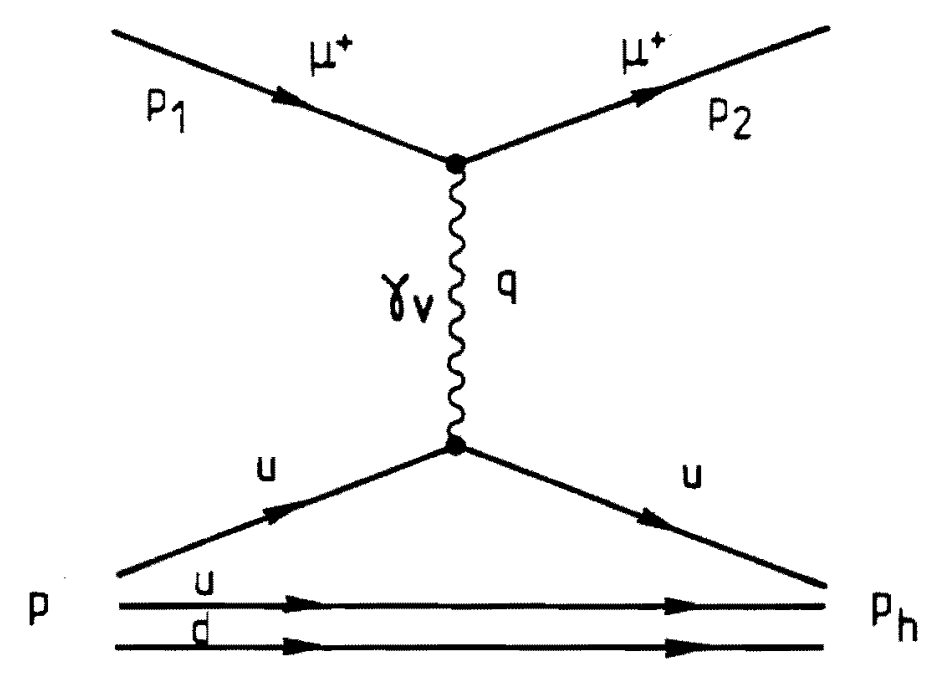

(b)

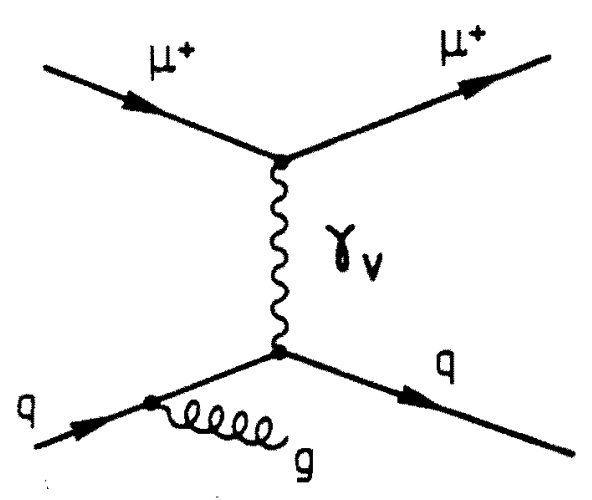

(d)

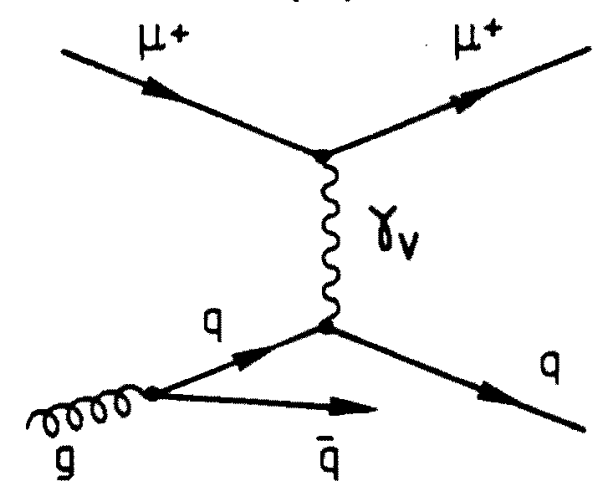

(c)

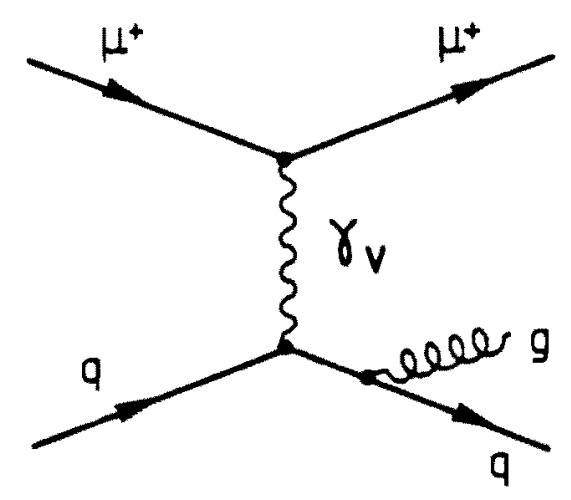

(e)

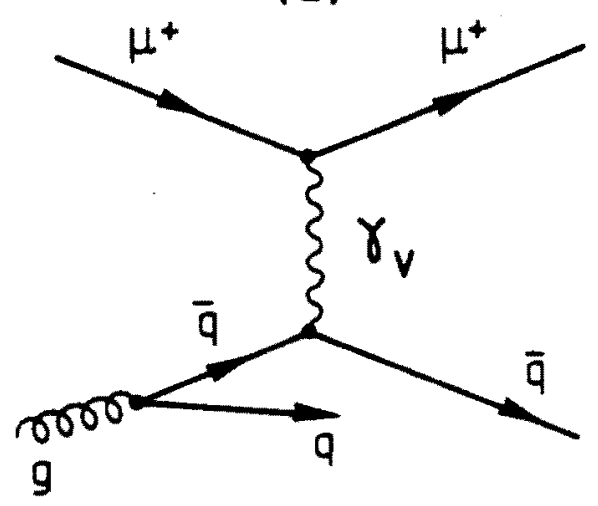

Fig. 4 


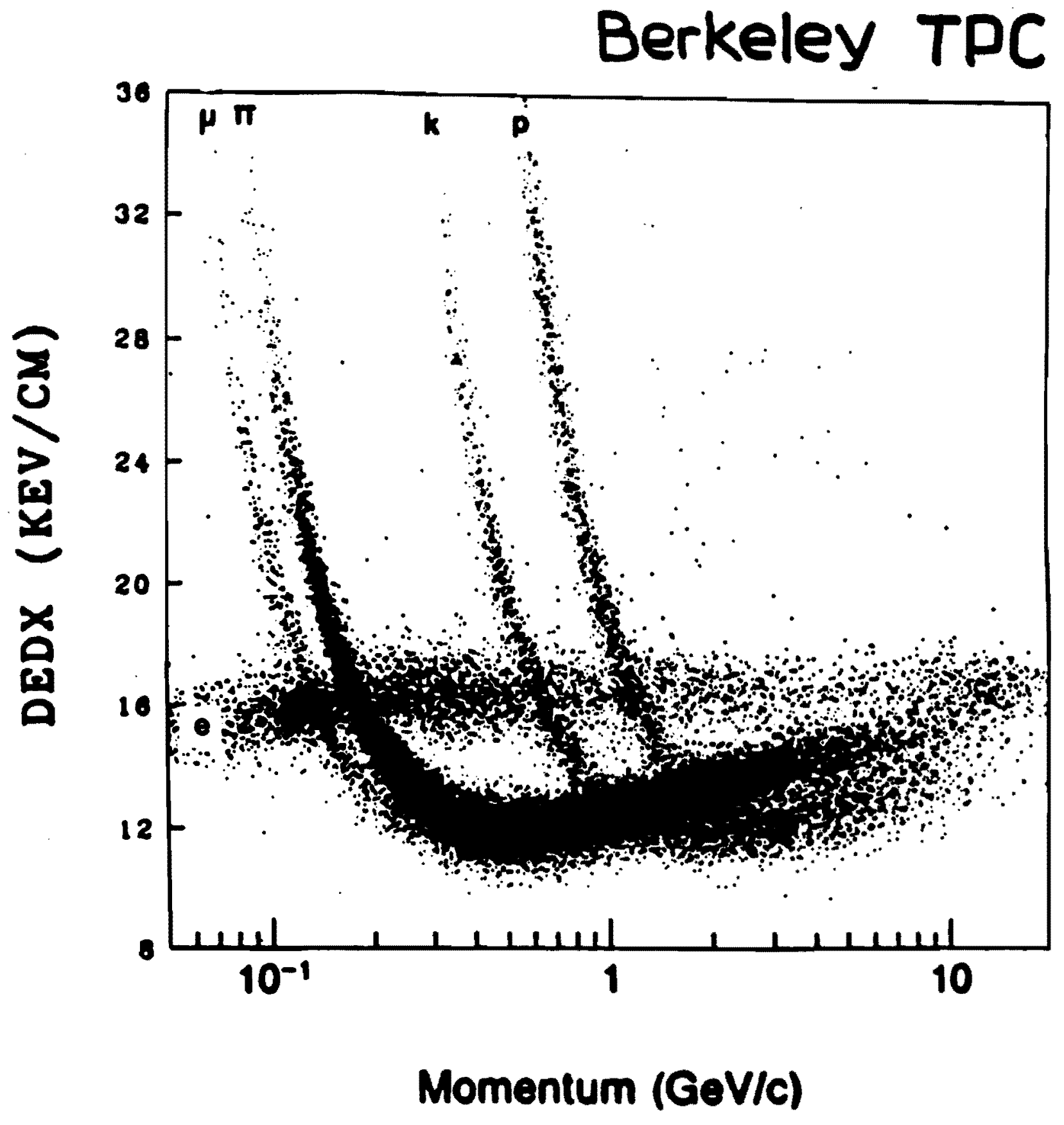

Fig. 5 


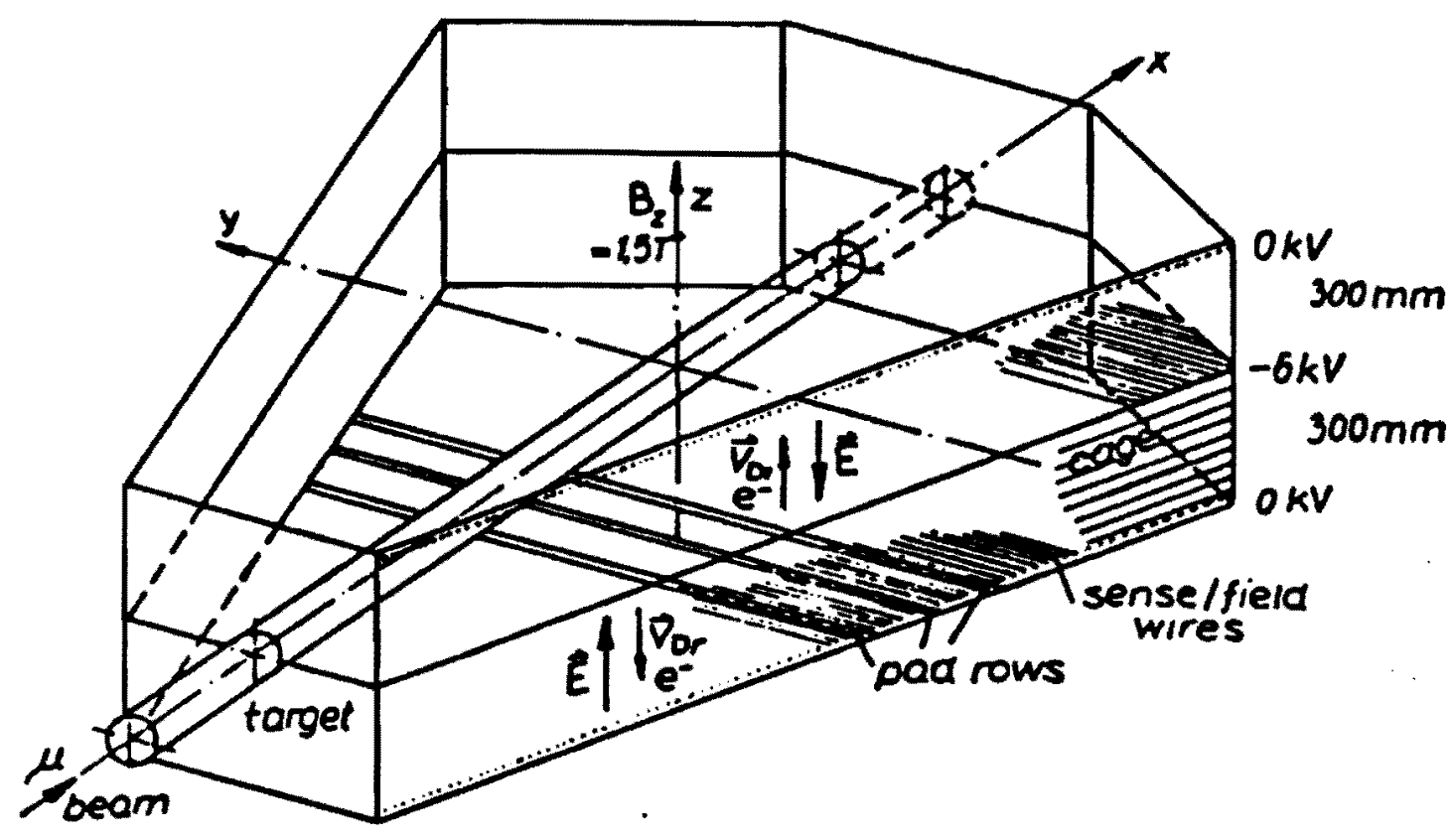

Fig. 6 Layout of the TPC field cage. The outer surface of the beam pipe is also covered with potential strips 


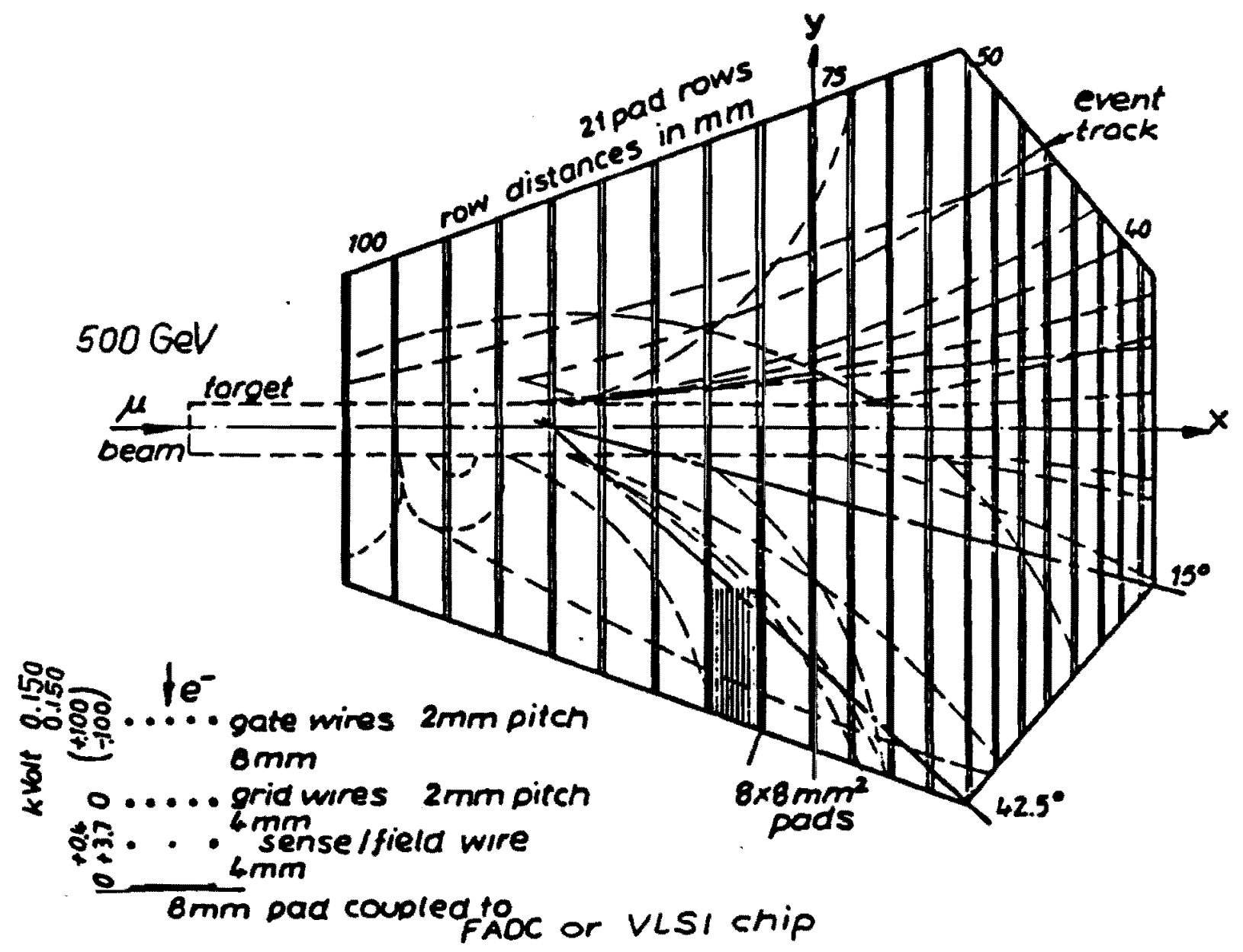

Fig. 7 Layout of the TPC end plate 


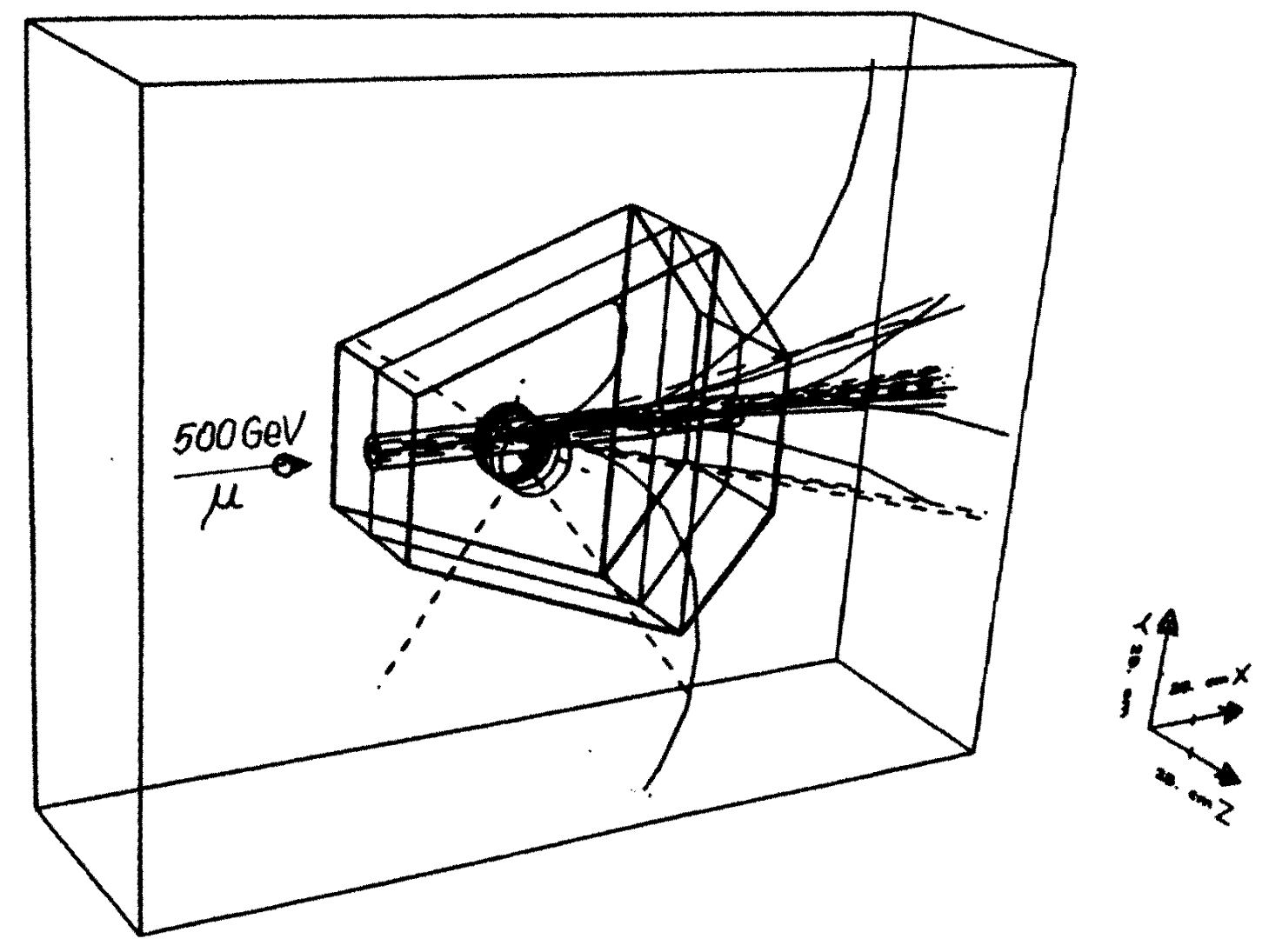

Fig. 8 


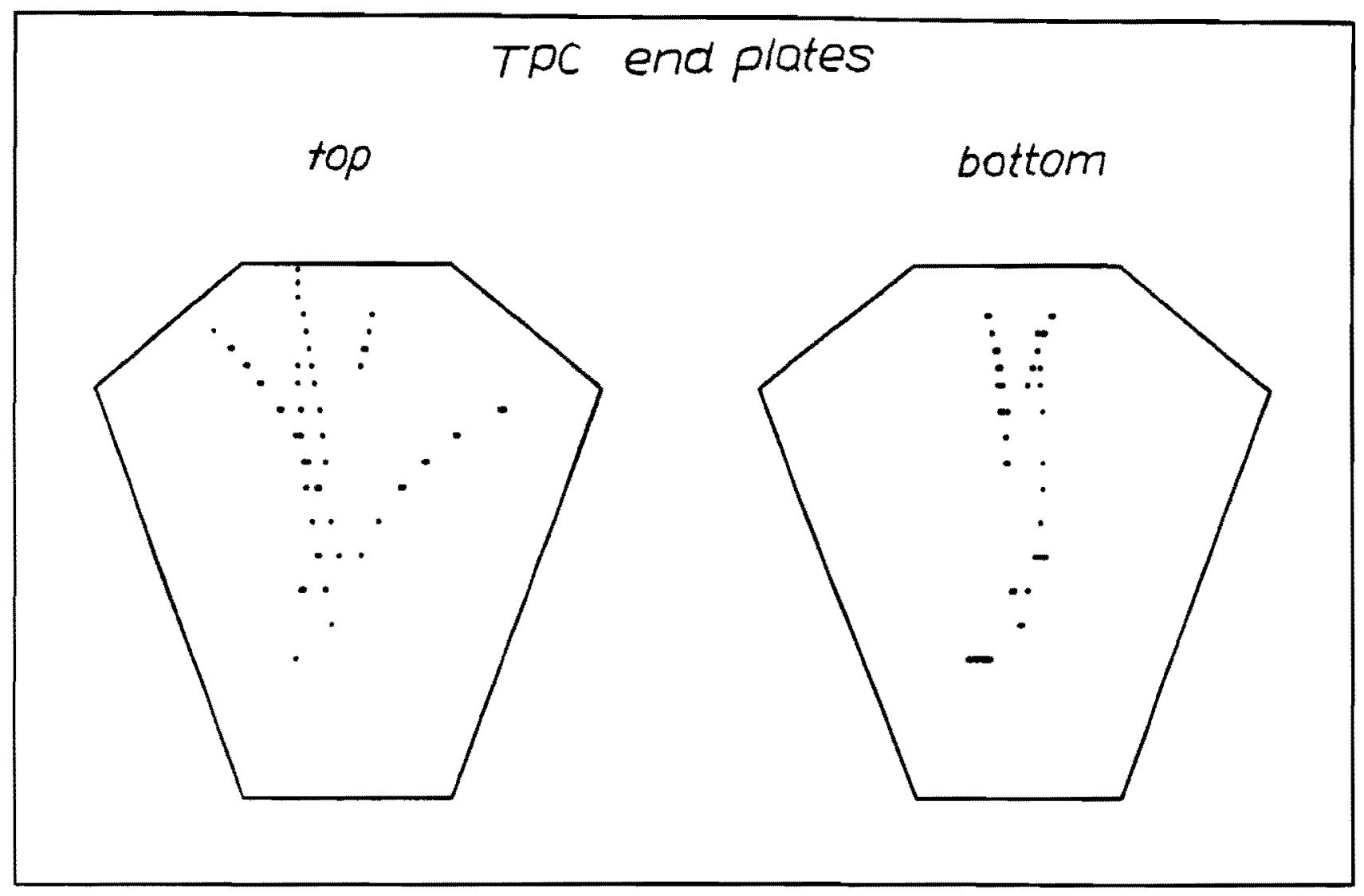
RUN
1. EVENT
1. PADS HIT
104

Fig. $9 a$

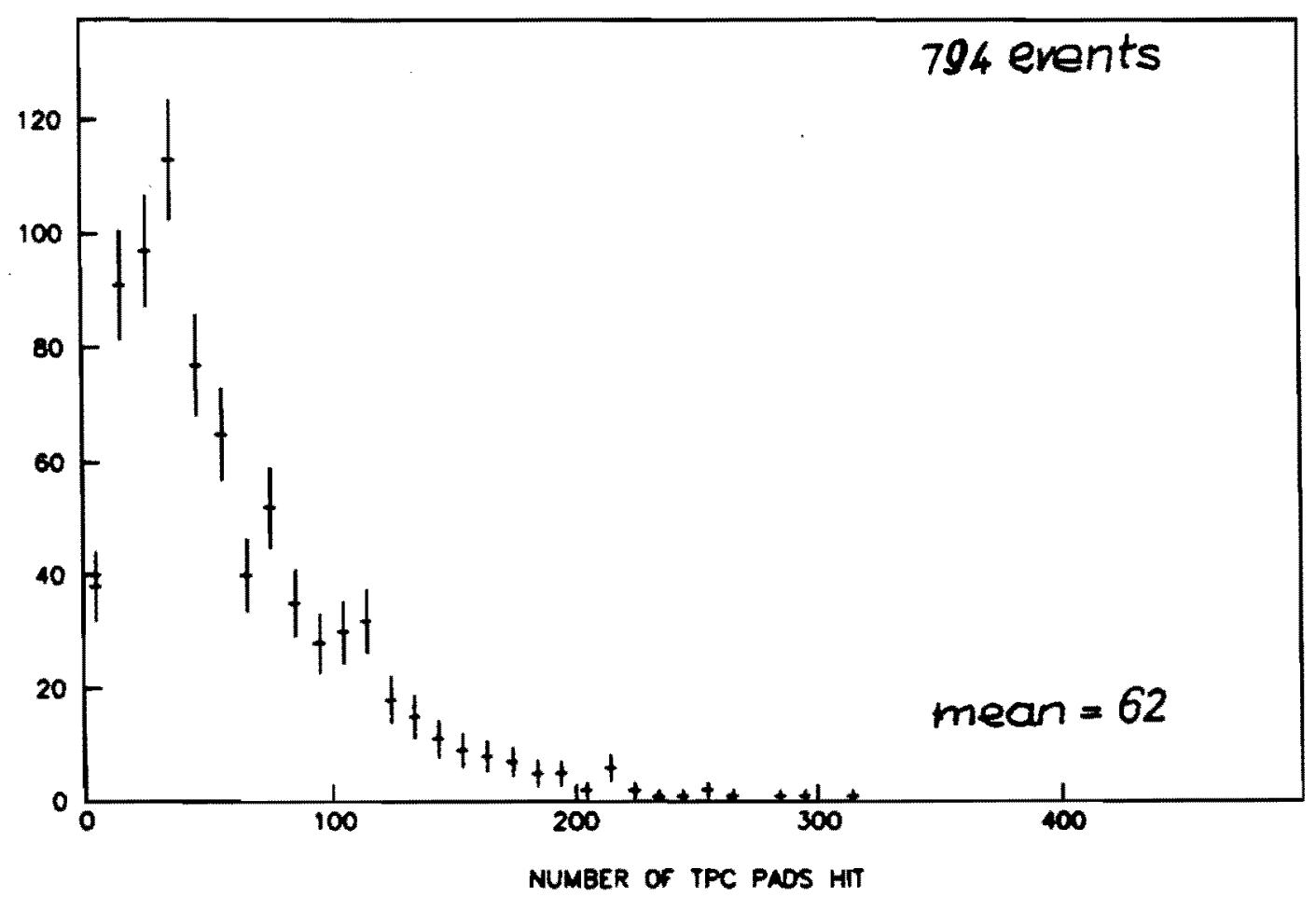

Fig. $9 b$ 
Fig. 10

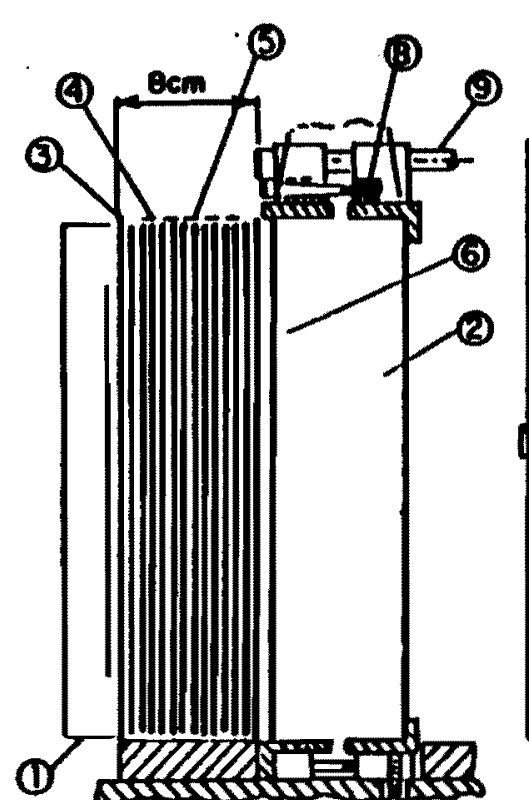

Section 'B-8' showing Du plates

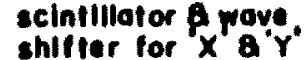
shifier

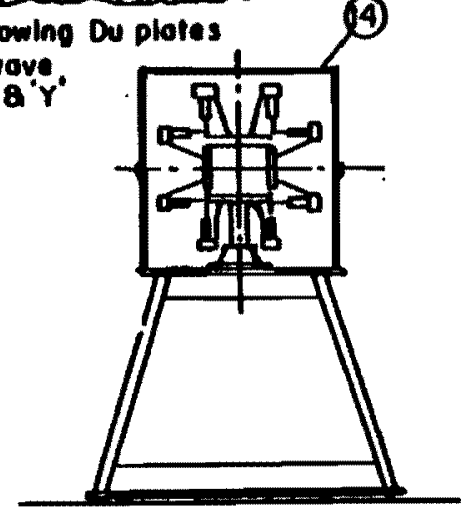

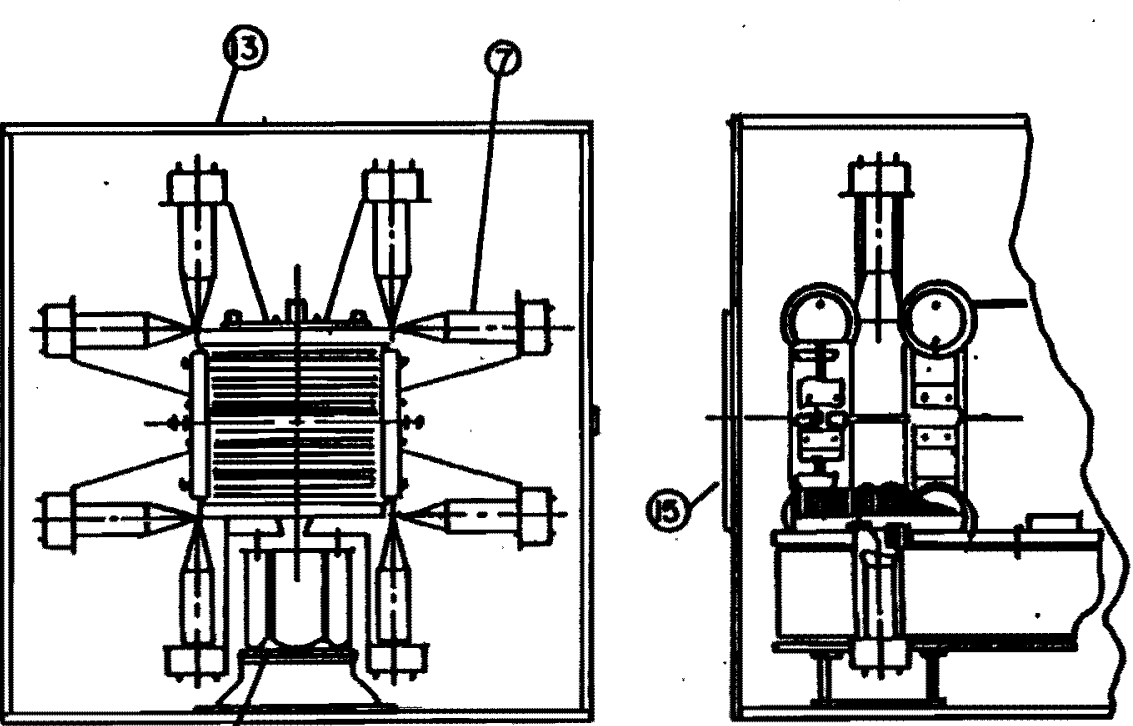

(1)

$4.7 \mathrm{~m}$
(1) X MOOULE ASSY.

(2) Y NODULE ASSY.

(3) DU SHEET 300m $30 \mathrm{Can} \times 3$ mon

(4) SCINTILLATOR $3 \operatorname{mon}$ THK. (12/M.)

(5) WUE SIFTER UGHT PIFE (2/M)

(6) GRAPHITE ENO PLATE

(7) PHOTOMULT. TUEE ASSY. (A/M)

(8) MOOULE CLAMP ASSY. (2/Mad)

9. MOOULE MTG. TENSION ROOS (3)

(O) SUPPORT BEAM ASSY.

(II) MOOULE ASSY'S (58)

13 CONNECTOR MTE. PLATE

(3) LIGHT TIGHT ALUM. BOX

(14) REMOVABLE TOP

(15) COATED IMLAR WNDOW (16) SUPPORT EASE ASS'Y

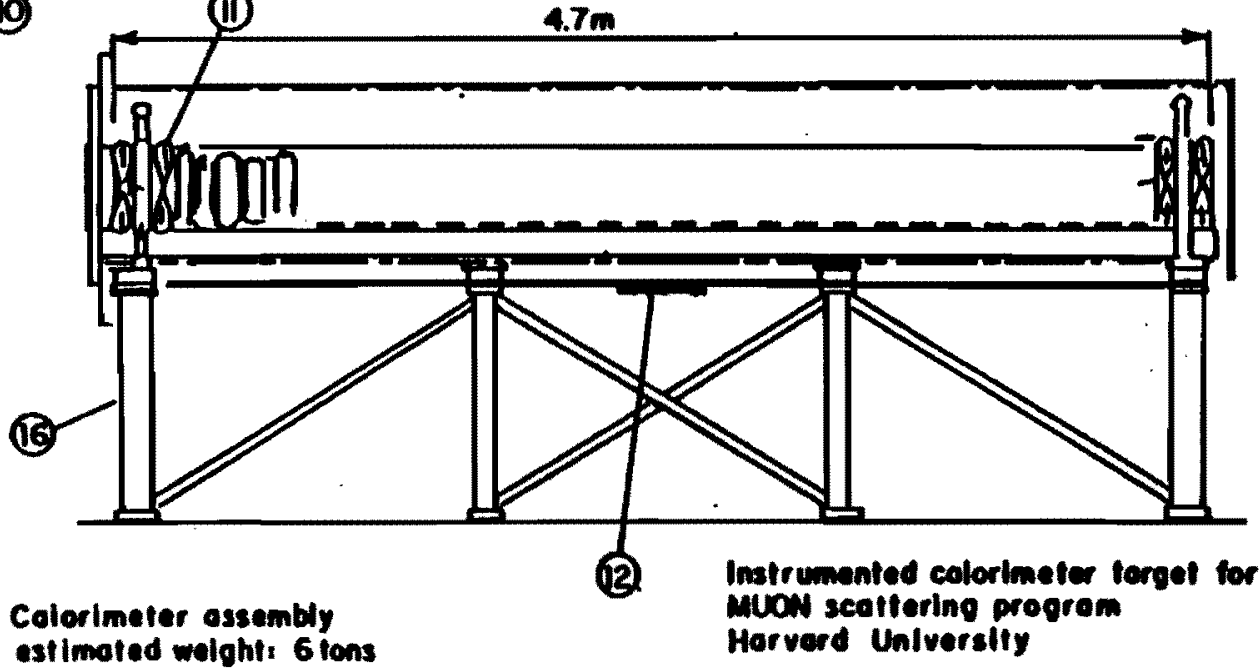


DEEP INELASTIC KINEMATICS

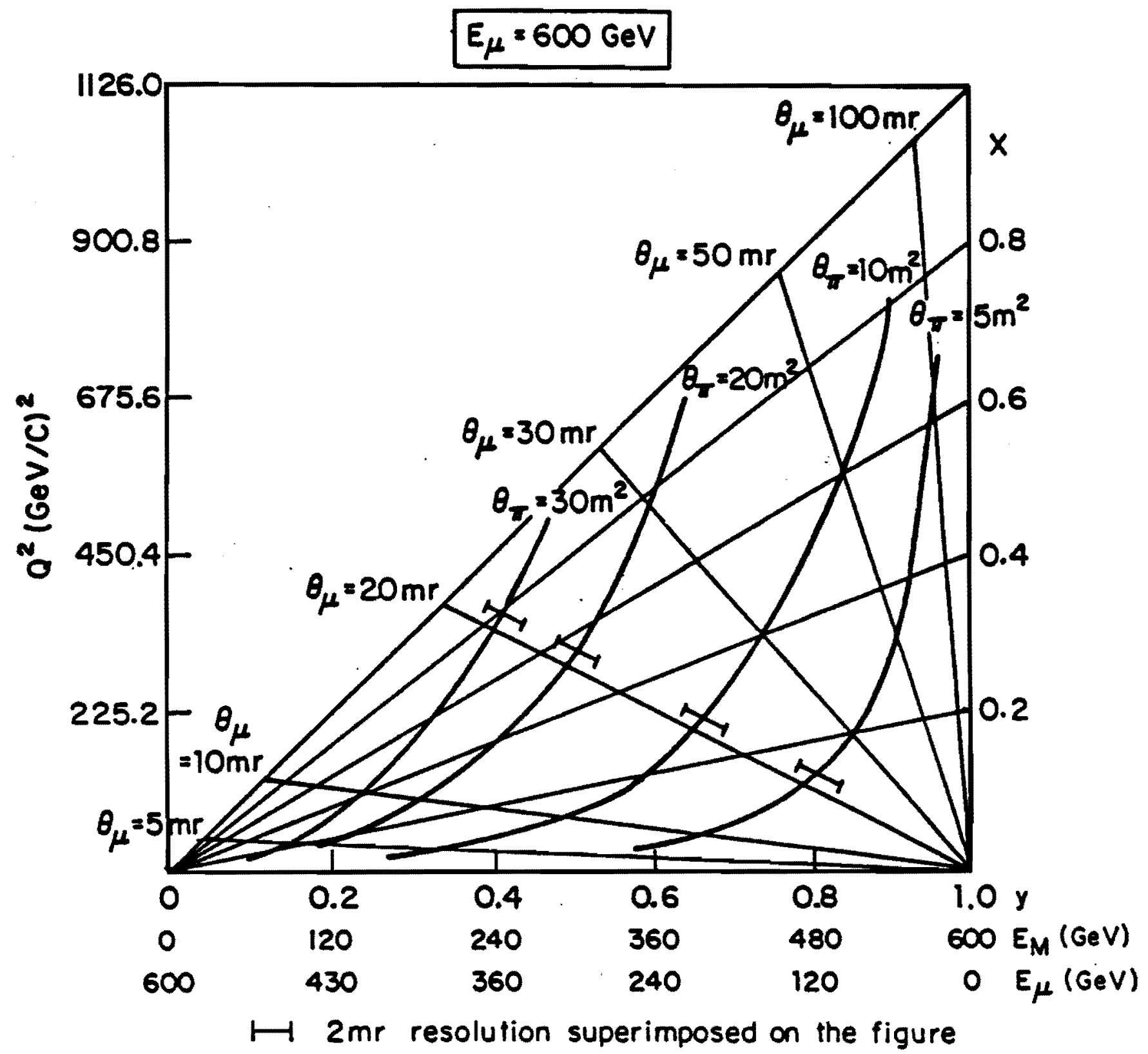

Fig. 11 